\title{
Mini-Micro-Mainframe Computer Marriage: Combining Technologies in a Radiology Results Reporting System
}

\author{
George David and Scott Gregory
}

\begin{abstract}
The minicomputer-based information system in the Department of Radiology at the Medical College of Georgia Hospital and Clinics was placed in service in February, 1982. This system represents a sizable investment in minicomputer hardware in addition to more than 6 years of software customization. One serious deficiency in the original system was the lack of a radiology results reporting facility. Several options were considered to provide the department with this capability. The most obvious option was retiring the existing system and replacing it with one of a number of commercial products already offering results reporting. In-house development of a reporting facility lent itself more readily to microcomputers than to the existing minicomputer system. Due to system customizetion, economic and time constraints, it was decided to merge an in-house developed microcomputer-based report module into our existing minicomputer system. The minicomputer was able to communicate with and transfer files to and from both micro and mainframe systems. Combining technologies allowed us to continue taking advantage of our sizable investment in money, time, and customization while providing a microcomputerbased report module. Radiology reports are now typed on microcomputer word processors and bulk transferred to the minicomputer. The minicomputer provides access to both unapproved and approved reports on system terminals throughout the department. It also enhances reports by merging patient demographics and registration information. Using existing communications facilities to the hospital mainframe system, reports are provided throughout the institution.
\end{abstract}

C 1989 by W.B. Saunders Company

KEY WORDS: Radiology, computers, radiology information systems, minicomputers, microcomputers, radiology reports.

$\mathbf{T}$ HE ORIGINAL information system in the Department of Radiology at the Medical College of Georgia Hospital and Clinics (MCG) was placed in service in February, 1982 operating on an IBM series/1 minicomputer. Software for this system, custom written for MCG by Charles May and Associates of Jacksonville, Florida, was primarily used to facilitate billing and to maintain patient examination histories. The original system operated on 512 Kbytes of memory and supported four registration terminals and one billing system terminal. Since going into service, the operating software has been substantially customized and enhanced by inhouse expertise and hardware and memory have been upgraded.

One serious deficiency in the original system was the lack of a radiology results reporting facility. A number of radiology report systems deal with the generation procedure. Jost ${ }^{1}$ discusses several such systems such as those based on mark-sense technology, touch-screen terminals, and telephone access. The latest innovation in the market is the voice-recognition system. ${ }^{2}$ Our goal, however, conformed to Jost's assertion that "most radiologists strongly prefer to dictate reports in the traditional manner." Thus the system was developed to preserve the existing dictation process as a transcription-oriented system stressing processing, archival, and distribution of the 350 to 400 reports generated daily.

Several options were considered in order to provide the department with such a reporting facility. Retiring the existing system and converting to a turn-key commercial product was rejected due to the high degree of customization on the existing system, the sizable investment in minicomputer hardware, and limited resources. Another option considered was to entirely rewrite the system to operate on a microcomputer network, allowing the use of commercial word processing and data base products that were not available at the time the original system was purchased. This approach was also rejected as an inefficient use of limited resources and time. It was also not clear that a microcomputer network would be able to match the performance of our Series/1, a machine that is a current supported product specifically designed for multiple users simultaneously operating various applications.

Rather than retiring the Series/1 or choosing between micro or minicomputers, we elected to

From the Department of Radiology, Medical College of Georgia, Augusta, GA.

Address reprint requests to George David, MS, Department of Radiology, Medical College of Georgia, 1459 Laney-Walker Blvd, Augusta, GA 30912.

(C) 1989 by W.B. Saunders Company

0897-1889/89/0201-0007\$3.00/0 
build a radiology report system by combining the two technologies. Technically this was accomplished by typing reports on a personal computer (PC) word processor and using file transfer capabilities between the Series/1 and the PC. Combing technologies allows us to take advantage of our sizable investment in money and time by continuing to use existing customized software while providing a timely and cost-efficient way of adding new applications such as word processing in which microcomputers clearly excel. The report system developed in this manner includes transcription, approval, short-term archival, printing, and distribution of radiology reports. It also ties into the hospital mainframe computer for electronic transfer of radiology reports throughout the medical center.

\section{METHODS}

Development of the report system centered around two commercially available software products, one a word processor, the other a terminal emulation program. A search for a personal computer word processor suitable for radiology transcription yielded a program called "WordProof" from IBM (Danbury, CT). WordProof has several attributes that make it appropriate and desirable for use in this application. It is a limited feature word processor that has word wrap and a spelling checker, both essential to transcription. Although it does not support block copies, move, delete, or have other features not used in the transcription of radiology reports, it is very fast, extremely easy to use, and contains an excellent spell-check program that includes user dictionaries. Report files created by WordProof are ASCII character strings with no embedded control codes. Files can therefore be straightforwardly transferred to and used on the Series/1 without modification.

An IBM program called "Intelligent Work Station" (IWS) (Danbury, CT), developed specifically for interfacing personal computers with the Series/1, was selected to perform terminal emulation. IWS causes the PC to emulate an IBM 3101 terminal as seen from the Series/1 minicomputer. In addition to terminal emulation, IWS also supports bidirectional file transfers between the PC and Series/1 and direct printing from the Series/ 1 to the $\mathrm{PC}$ printer. The PC is connected to the Series/1 through a standard RS232interface.

In moving the transcription process from typewriters to microcomputers, radiologists faced no operational changes in the report generation process except for the option of using canned reports. As before, reports are dictated by radiologists on mini-cassette tapes. Before implementation of the computerized system, reports were transcribed directly onto multipart request forms that were signed, manually torn apart, and distributed. Transcriptionists now type into a word processor and, instead of immediately printing each report, save all reports from a single cassette (typically 10 to 20 ) on a clear
PC diskette. Each file is checked for spelling errors before storage.

Each registration on the Series/ 1 is assigned a two character code. This code in combination with the patient number uniquely identifies the registration in the following way. The code is actually a hexadecimal number between 1 and 256, permitting patients to have up to 256 reports on file simultaneously. Since the system was designed to be a short-term archive of approximately 2 weeks, this is normally not a limitation. While the two character code may be reused for a particular patient after a period of inactivity, at any particular time it uniquely identifies the encounter. A registration or encounter code may represent one or several radiological examinations. Each report is assigned a DOS file name consisting of the six digit patient number followed by the two character registration code and the first three characters of the last name as the file extension. This three character extension serves as a validity check. The patient number and registration code allow one to reference information about the patient and the examination entered on the Series/1 at registration. This information includes name, sex, race, date of birth, examination date, referring clinic, and referring physician.

After typing an entire cassette tape on a diskette at microcomputer transcription stations, the diskette is carried to a microcomputer dedicated report transfer unit where all reports on this diskette are bulk transferred to the Series/1 minicomputer using IWS. Using a dedicated report transfer unit avoids transmission delay problems for transcriptionists. During transfer of reports, transcriptionists return to their stations and begin work on the next cassette tape (Fig 1).

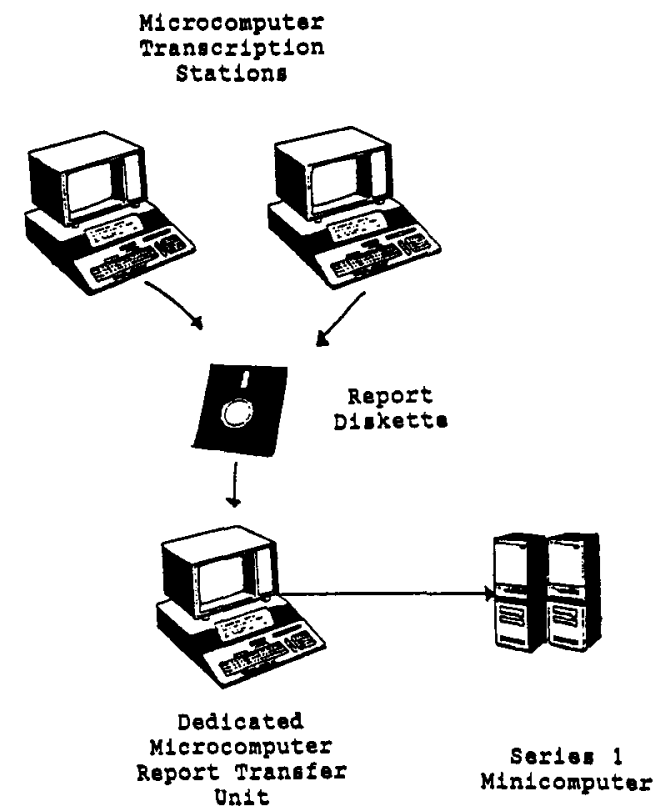

Fig 1. Using microcomputer transcription stations. An entire dictated cassette tape is typed to a report diskette. Diskettes are carried to a dedicated microcomputer report transfer unit where they are bulk transferred to the Series/ 1 minicomputer. 
Because each diskette reflects reports contained on a single radiologist's tape, the transcriptionist, dictating radiologist, date of dictation, and date of transcription are the same for each report on the diskette. Rather than requiring transcriptionists to type this information on each report, it is entered only once at the time of transfer and automatically assigned to each report on the diskette. Before files are transferred, file names are checked for validity and the transcriptionists are given the opportunity to make file name corrections. Once file names are resolved, the transfer of reports continues without further operator intervention. After all reports are transferred, approval copies are automatically printed on the PC printer.

As previously described, each report is divided into four sections (Fig 2). The top five lines of each page form a header that is automatically added to each report by the Series/1. Following the header, specific information on the patient and registration is automatically added by the Series/ 1 to each transcribed report. The body of the report follows and is the only part typed from dictation and saved on diskette. Finally, a footer at the end of each report identifies the transcriptionist and dictating radiologist (supplied by transcriptionists when reports are transferred to the Series/1) as well as referring physician (from registration). Pagination and the handling of headers and footers on multi-page reports are handled by the Series/1.

The report approval process required minor operational changes for radiologists. As had been done previously, a stack of report approval copies corresponding to a single dictation tape is distributed to the dictating radiologist who indicates approval or changes. If changes are indicated by the radiologist, reports are modified by editing the original PC report diskette using WordProof. Any correction to a report causes the directory time/date stamp on the PC report file to be

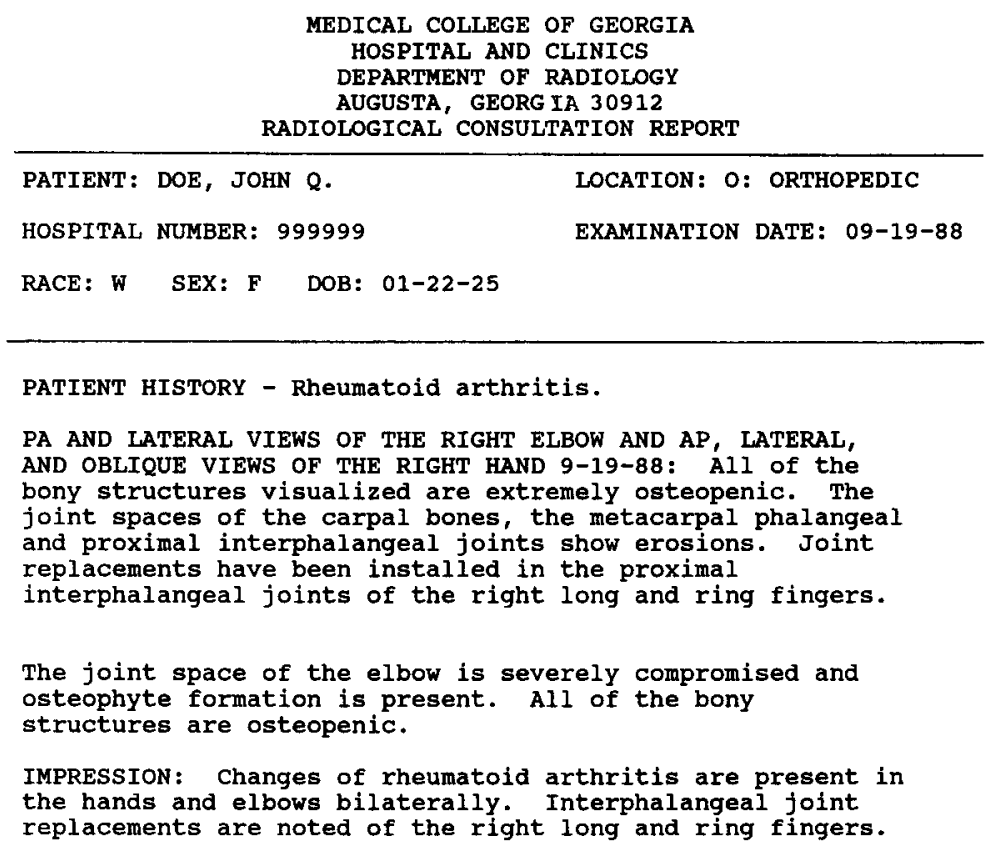

Fig 2. Sample report merging data from micro- and minicomputer. Only the center body of report was typed by transcriptionist.
CLARENCE JOE, M.D., ATTENDING RADIOLOGIST
DICTATION DATE: 09-19-88 TRANSCRIPTION DATE: 09-23-88 TRANSCRIPTIONIST: JH (01)
REQUESTING PHYSICAN : NONE PROVIDED
PAGE \#1 
updated. Because reports are submitted, printed, and reviewed based upon the dictation tape, reports are approved on the minicomputer by resubmitting the original $P C$ report diskette to the Series/1 on the microcomputer dedicated transfer unit. During the approval process, the Series/1 checks the time/date stamp of each report file. Any reports that have been modified since the submission of the unapproved copy are retransmitted to the Series/1 in corrected form and new approval copies are generated.

Approved reports are flagged for subsequent printing of distribution copies on the Series/1. In order to take advantage of the ability to print reports in sorted order, newly approved reports are bulk printed on the Series/1 twice a day. Three different groups of reports are printed, one for inpatients, one for outpatients, and one for referring physicians who have requested copies. Each group has its own unique sort to facilitate distribution.

Twice daily newly transcribed and newly approved reports are bulk transmitted to the hospital's host computer system from the radiology minicomputer. The same interface used for billing is used for report transfer, an IBM "Remote Job Entry" (RJE3780) (Danbury) protocol on a four-wire direct connected bisynchronous line. Like the radiology information system, MCG's hospital-wide information system is supported and custom developed in-house. Each report transferred by radiology to the hospital host system is accompanied by information identifying date of service, dictating radiologist, and requesting physician. The host system maintains its own long term archive of reports and allows users throughout the institution access by patient number.

\section{DISCUSSION}

Separating word processing from the Series/1 and combining mini, micro, and mainframe computer technologies affords several operational advantages. Because transcription is done on individual PCs, this work proceeds independent of mini and mainframe computer schedules and need not be interrupted when these systems are down for back-up or maintenance. The PC diskette serves as back-up to the report files transferred to the Series/ 1 and is not re-used until its reports have been printed and distributed.

The choice of commercial PC word processing software allows a great flexibility in the selection of software for transcription. WordProof is easy to use and can be learned in a short period of time. At a cost of approximately $\$ 50$ per station, it is also considerably less expensive than most minicomputer software.

By basing the report system on the dictation tape, minimal changes were made in the radiologists' dictation and approval protocol. By relating each diskette to a cassette tape, transcriptionists have an approach to keeping track of information that is a logical extension of previous practices. Using a separate PC terminal allows report file transfers to take place in the background while new reports are being transcribed, minimizing delays in operations.

Storing reports on the minicomputer permits on-line inquiry on any of ten departmental inquiry stations as well as batch transmission to the hospital's host system. The minicomputer automatically merges registration information into each report making reports more complete and eliminating redundant data entry by transcriptionists. Bulk printing of reports on high speed printers in sorted order has significantly reduced manual work associated with report printing and distribution.

Mixing micro, mini, and mainframe computer technology has proved advantageous in that the best features of each system are exploited. This combination allows the Medical College of Georgia to use the microcomputer market in its information system without losing its investment in minicomputer hardware and software.

\section{REFERENCES}

1. Jost GL, Trachtman J, Hill RL, et al: A computer system for transcribing radiology reports. Radiology 136:6366,1980

2. Rosen RA: X-ray reports faster and cheaper. Applied Radiology 17:18-19, 1988 